\title{
REVERSE ZOONOSIS AND ANIMAL HEALTH
}

\author{
Kashif Hussain ${ }^{* 1}$, Muhammad Ijaz ${ }^{2}$, Ameer Hamza Rabbani ${ }^{3}$, Ahmad Ali ${ }^{1}$ and Yasir Razzaq Khan ${ }^{1}$ \\ 'Department of Medicine, Cholistan University of Veterinary and Animal Sciences, Bahawalpur \\ ${ }^{2}$ Department of Veterinary Medicine, University of Veterinary and Animal Sciences, Lahore \\ 3Department of Surgery, Cholistan University of Veterinary and Animal Sciences, Bahawalpur \\ *Corresponding author: kashifhussain@cuvas.edu.pk
}

\section{INTRODUCTION}

When pathogens are capable of transmission from human to non-human hosts, such phenomenon is referred as reverse zoonosis. It is also known as anthroponosis or zooanthroponosis (Hubálek 2003). Whereby, Anthroponosis is a term used to describe human to nonhuman animal transmission, as well as human transmission, zooanthroponosis is exclusively used to explain human to animal pathogenic transmission (Joint and Organization 1967; Hubálek 2003). Humans transmit the pathogenic agents to the animals, where they sometimes mutate and spread through different species of animals. Depending upon the nature of pathogens, reverse zoonosis is categorized as reverse zoonosis by bacteria, viruses, protozoa/parasites and fungi. Infection potential of a pathogen in a population, either humans or animals, depends upon the mode of its transmission. Direct human to human, animal to human or human to animal via aerosol or feco-oral route is easy and quicker than the involvement of any other vector. This is because in a geographical distribution, presence and survival capabilities of different vectors are different. If a vector is abundantly present in an environment, then disease spreading rate will be higher and arthropod vectors are the most notorious in this field. Attempts have been made to compile the incidence of reverse zoonosis occurred around the world. Most of the focus is on the bacterial and viral pathogens, as they are mainly involved in interspecies transmission. Since transmission of pathogens is either direct or vector involved, both of these aspects are considered in discussion. Human pathogens can affect variety of species of domesticated animals, as well as wildlife. Evidence of reverse zoonosis can be found in domestic animals or captive wildlife, but it is difficult to ascertain in the free-range wild animals (Fig. 1). It is also observed that drug resistant pathogens are also involved in interspecies transmission, which is alarming. Mutation is also one of the factors in the reverse zoonosis that is discussed in this chapter.

\section{Reverse Zoonosis by the Bacterial Pathogens}

Bacterial zooanthroponosis has been documented since long, affecting wide range of animals including farm animals, companion animals and the wild life. The mechanisms involved in the transmission vary among various species of animals. However, generally the mechanisms involved include the direct contact, feco-oral route, fomites, direct inoculation and aerosol route (Hackendahl et al. 2004; Messenger et al. 2014; Barasona et al. 2017). There are certain bacteria which are involved in the reverse zoonosis, causing the outbreak of diseases of human origin in animals with possible outcome of severe ailment, drug resistance and mortalities.

\section{Methicillin-resistant Staphylococcus aureus (MRSA)}

Staphylococcus aureus (S. aureus) is considered as a bacterium of substantial importance in human medicine. It is also proclaimed as a serious public health concern. $S$. aureus imparts adverse effects and conditions in human beings, ranging from minor skin and soft tissue infection to serious ailments including meningitis, bacteremia and pneumonia (Tong et al. 2015). The non-human hosts (mostly livestock) are focused as the reservoirs of infections and antibiotic resistance related to humans. Now, the interest has been shifted to investigate the role of humans as the source of infection for animals (zooanthroponosis) (Fluit 2012; Price et al. 2012).

Anthrozoponosis is more common than the zooanthroponosis by the $S$. aureus. However, it is now obvious from the studies determining the potential of $S$. aureus to shift the host species, with resultant adaptation in new host and further transmission in newly adopted host species (Shepheard et al. 2013).

This phenomena of adapting in the new host after switching and interspecies transmission is now very well known to occur between humans and animals. Therefore, $S$. aureus associated with the human origin causes rapid colonization and infection in the host as compared to non-human primates (animals), either domesticated or in the wild (Schaumburg et al. 2012).

The premiere driver of host adaptation following the inter-host transmission is addition or deletion of the genes directly associated with mobile genetics of the bacterium (Spoor et al. 2013) . Therefore, non-human host provides suitable environment to the bacterium to acquire the novel virulence and antimicrobial resistance (Sung et al. 2008). The antimicrobial resistance and acquisition of unusual virulence have been observed in the clones of MRSA from humans that have been reported in livestock and vice versa (Sung et al. 2008; Köck et al. 2013). Reverse zoonotic potential of the $S$. aureus is adversely affecting many livestock species. One such example is from the equines, where 11 horses from 


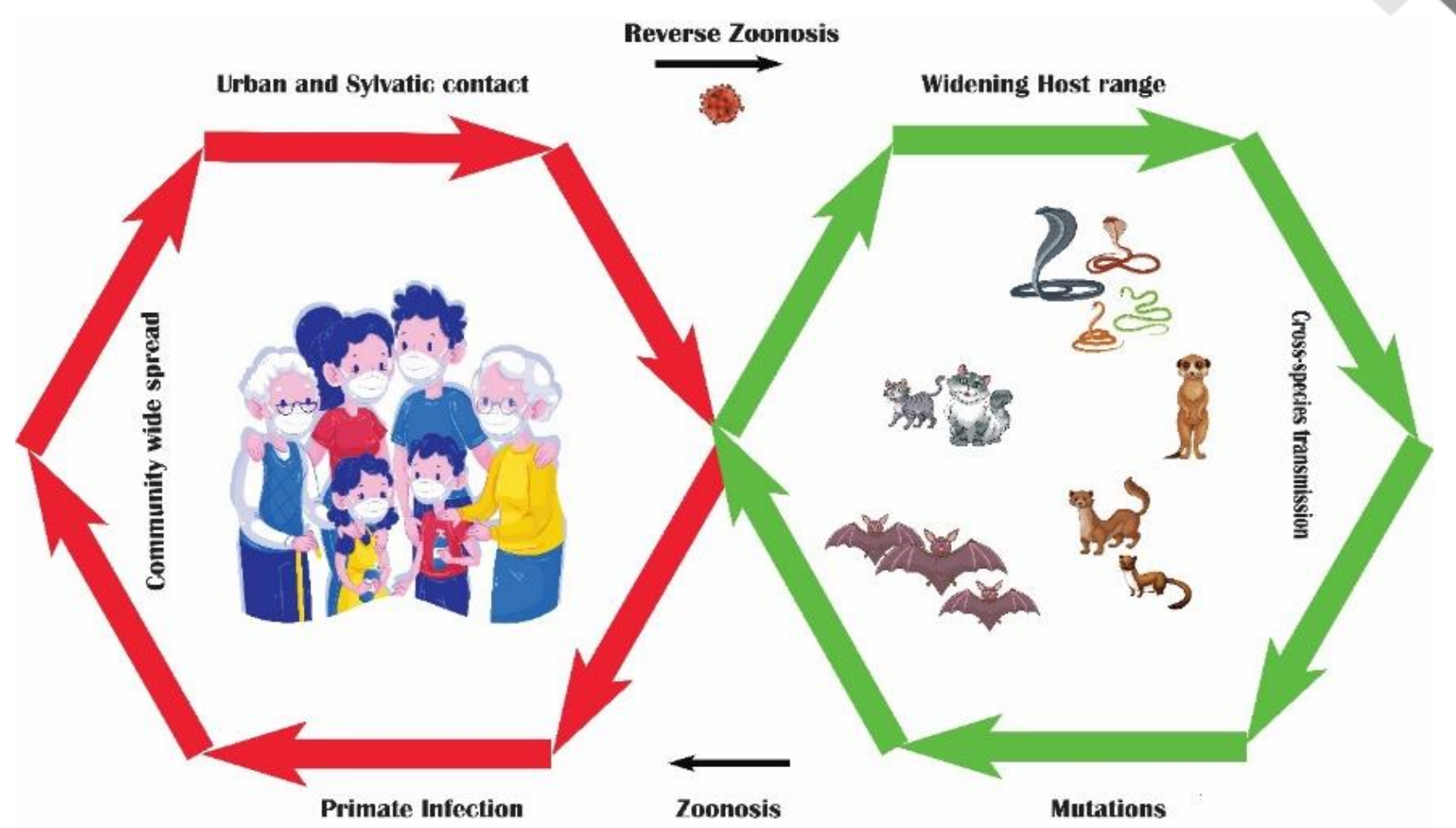

Fig. 1: Showing transmission possibility of pathogens between human and animals, mutation and it's spread through community

different farms exhibited MRSA infections. As the MRSA is extremely rare in horses, the outbreak was acquired at the hospital, which is known as nosocomial infection (Seguin et al. 1999). However, potential of the $S$. aureus to affect non-human primates is not restricted to equines, as it also affects other species like cows, turkeys, and pigs (Hasman et al. 2010; Price et al. 2012). Although, mutation is continuously reducing the zoonotic threat for humans, but it augments the $S$. aureus resistance capabilities, which will ultimately enhance the occurrence of MRSA infections (Price et al. 2012). Human to livestock transmission of MRSA has also been documented in advanced countries of the world,including USA (Smith 2015), UK (Graveland et al. 2011), Canada (ArmandLefevre et al. 2005), China (Zhou et al. 2018), Germany (Köck et al. 2009) and France (Price et al. 2012).

\section{Tuberculosis}

Bovine tuberculosis (TB), caused by the Mycobacterium (M.) bovis, $M$. tuberculosis, or rarely by $M$. caprae, is an important and significant disease of public health concern due to its zoonotic potential and resultant considerable economic losses (Torgerson and Torgerson 2010, Bayraktar et al. 2011a, 2011b). In humans, majority of the TB cases happened either due to utilization of the contaminated milk or through aerosols from the infected animals (Moda et al. 1996). However, situation has become complex with the emergence of reverse zoonosis. Sharing of the micro environment and premises by the human and non-human primates has significantly potentiated the disease (Shitaye et al. 2007). Previously, it has been reported that humans are preliminary the cradle of $M$. tuberculosis infection for animals (BhanuRekha et al. 2015). It is important to consider that M. bovis can infect urogenital system in humans and secretions from such patients can affect the animal health through reverse zoonosis (Ocepek et al. 2005). An extraordinary pervasiveness of zoonotic $\mathrm{TB}$ in humans is due to its significantly high occurrence in the cattle (Asiak et al. 2007). Nonetheless, transmission of the TB can occur mutually and previous studies have supported this phenomena of reverse zoonosis, where isolation of $M$. tuberculosis has been done from cattle and that of $M$. bovis from humans (Ocepek et al. 2005; Jenkins et al. 2011; Adesokan et al. 2012). The canvas of TB is expanding and many animal species other than the cattle have also been affected by the human infection. Lesions of TB have been reported in wild red deer and boars, where they had been confined under the intensive care management conditions such as large game fences and auxiliary feeding locations (Schöning et al. 2013). Spread of the same strains of TB found in humans to these wild deer and boars suggests that the possible spread was either through direct human contacts or their excretions (Barasona et al. 2017). The reverse zoonotic potential is also affecting the free range wild life species and in such an incidence the necroscopic findings of the African elephant revealed seriously damaged lungs due to the strain of the $M$. tuberculosis. This reverse zoonosis phenomenon was the culprit behind this infection that might be due to aerosolization of the agent either 
through contaminations by the surrounding human community or tourists (Miller et al. 2019). There is significant evidence of direct or aerosol transmission of the $M$. tuberculosis from humans to companion animals (Hackendahl et al. 2004).

\section{Escherichia coli}

Multidrug resistant strains of pathogenic Escherichia (E.) coli are important in public health concern because of their speedy spread through the large population and also due to their supposedly perpetual evolution (Ewers et al. 2010). Transmission of the zoonotic pathogens has increased between the humans and animals due to sharing of the same habitat. There is strong evidence of transmission of E. coli from humans to livestock including mountain gorillas, which revealed that habitat sharing is affecting the human to animal transmission of this pathogen through the reverse zoonosis (Rwego et al. 2008). Escherichia coli DSM22664 is a human pathogen which showed the similarity index of $>85 \%$ with six $\mathrm{B}_{2}-$ O25b-ST131 CTX-M-15 strains detected in animals in five different countries of Europe. This is an indication of its potential of distant transmission (Nicolas-Chanoine et al. 2008). The possible spread of virulent serotypes of cephalosporin-resistant $E$. coli from human to companion animals, especially dogs, has been previously investigated in Germany (Ewers et al. 2010). These strains were previously considered exclusively for human population but emergence of the CTX-M-15-positive $\mathrm{B}_{2}-\mathrm{O}_{25}: \mathrm{H}_{4}-\mathrm{ST}_{13}$ strain in a dog infection is alarming (Coque et al. 20o8; Nicolas-Chanoine et al. 2008; Pomba et al. 2009). Presence of the clonally related human pathogenic multiresistant strains of E.coli in the pet animals across different European countries has pointed out its potential of inter-species transmission, humans to other animals and vice versa (Ewers et al. 2010).

\section{Helicobacter pylori}

Helicobacter pylori is an important pathogen of human gastrointestinal tract, causing serious and prolonged ailments. Animals in the captivity are also among those affected by this pathogen of human origin through reverse zoonosis phenomena. In such an example in Australia, the stripe faced marsupial suffered repeated and multiple outbreaks of the $H$. pylori while under captivity; the causative strain had $100 \%$ association with the strain of the human origin. Transmission of $H$. pylori to these animals was from the handlers of these animals, which further emphasizes the importance of the reverse zoonosis (Every et al. 2011).

\section{Salmonella spp and Shigella spp}

Salmonella and Shigella have significant potential to cause morbidity and mortality in captive gorillas (Stetter et al. 1995; Mundy et al. 1998). It has been found that freerunning mountain wild gorillas are affected by these bacteria in human adjusted territories. Some of the
Shigella species isolated from these wild creatures are $S$. sonnei, S. boydii, and S. flexneri (Nizeyi et al. 2001). These were found in sub-adults and grown-up gorillas aged from 6.0 to 11.9 years. The sharing of Salmonella spp. diseases among humans and gorillas has increased during previous years. The isolation of Shigella spp. interestingly from mountain gorillas may show upgraded reverse zoonotic transmission (Nizeyi et al. 2001).

\section{Transmission of Zoonotic Viral Diseases}

Viral infections such as dengue fever, yellow fever and zika fever, which mostly spread through arthropod vectors, can be justified by both anthroponosis and reverse zoonosis/zooanthroponosis, provided which host (human or mosquito) is considered the primary source of infection (Alekseev and Dubinina 2001). Conversely, infections such as Human Immunodeficiency Virus (HIV) which instigated in primates (LeBreton et al. 2007) and influenza virus attributed to avian species (Nelson and Vincent 2015), despite being originally identified as cases of zoonotic transmission, can now be termed as anthroponosis. Certain important viral pathogens of reverse zoonotic importance have been discussed in this chapter. Ironically enough, wild animals rarely come into direct contact with humans and due to their improbable presence in close vicinity, most cases of reverse zoonosis emerge between humans and wild animals as a consequence of contact with abiotic substrate contaminated with sapronotic agents (Hubálek 2003).

\section{Arboviruses}

Arthropod-borne viruses are called as Arboviruses. Several of these viruses, namely Zika virus, Dengue fever virus and Yellow fever virus belonging to family Flaviviridae, exhibit reverse zoonosis. Moreover, Chikungunya virus, which has been classified into Alphavirus genera, is also considered an arbovirus (Kuno et al. 1998; Forrester et al. 2012). These arboviruses have been reported to involve urban, as well as sylvatic transmission cycles. In case of Dengue fever, the virus has been known to jump from human population to primates through the help of Aedes aegypti mosquitoes. Similarly, certain arboviruses endemic in wildlife populations have the potential to infect human hosts (Figueiredo, 2019). Therefore, consequent intermingling of sylvatic and urban cycles is quite common due to a myriad of mosquito species capable of viral transmission.

\section{Zika Virus Fever}

Aedes mosquitoes are responsible for the spread of this single stranded RNA virus in human and other animal populations (Nah et al. 2016). In 1947, Zika virus was isolated from primates in Uganda, West Africa. In following years, most of the outbreaks of Zika virus were seen in temperate regions of Asia and Africa. The virus, for the most part, cycled between monkeys and arboreal mosquitoes but instances of human infections were 
sporadically reported. Zika virus experiences propagation in distinct sylvatic and urban cycles. However, in most cases zone of disease emergence for humans are areas where these cycles could overlap and co-exist. Previous investigations have postulated possible involvement of 47 animal species belonging to three orders (reptile, avian and mammal), other than primates and arthropod vectors (Singh et al. 2016). Another animal model studied antigenic response of Zika virus in pregnant rhesus macaques. To this end, Zika virus isolates from confirmed human cases were inoculated into pregnant rhesus macaques, resulting in propagation of viral bodies and detection up to 105 days after administration corroborating the probability of reverse zoonotic transference of viruses (Coffey et al. 2017).

\section{Yellow Fever}

Yellow fever is a flavivirus disease transmitted by arthropod vectors. It is an endemic to tropical regions of Africa and South America despite ample availability of vaccine. The spread of this disease across continents separated by vast oceans of water has been deliberated for several years now (Monath and Vasconcelos 2015).

However, Yellow fever is generally believed to have been introduced into American continent from West Africa approximately 400 years ago (Chippaux and Chippaux 2018). The disease was first reported amongst Europeans involved in the illicit slave trading business (Hamrick et al. 2017). It has been hypothesized that most probable source of the Yellow fever on the shores of new world may be attributed to the arrival of shackled infected individuals from West African countries, who were subsequently bitten by indigenous mosquitoes, thereby spreading virus to other humans upon next feeding (Yen et al. 2018). Regarding the concept of Reverse zoonosis, hypothesis suggesting anthroponosis of Yellow fever virus to un-infected Aedes species after feeding on diseased human is extremely noteworthy (Goenaga et al. 2012). In recent years, Yellow fever has been reported in nonhuman primates and other sylvatic communities living closely with un-vaccinated human populations (Figueiredo et al. 2018). An example of such a scenario was reported in Espirito Santo, Brazil, where PCR and immune-histologic examinations were performed on 22 deceased non-human New World primates and Yellow fever lesions were detected in 21 primates (Fernandes et al. 2017).

\section{Chikungunya Virus}

Since its emergence in Asia during 1950s, Chikungunya virus (CHIKV) has caused severe, debilitating arthralgia, with severe morbidity in humans. It is believed that this disease probably spread across Europe and the Americas due to genetic mutations that resulted in the development of a protective glycoprotein envelop (Tsetsarkin et al. 2016). These revelations led to the rational conclusion that Asian strain of the virus propagating in urban cycles originated from ECSA progenitor, which was introduced in the environment 50430 years before 2000 (Volk et al. 2010).

Chikungunya virus, being a single stranded RNA alphavirus commonly referred as arbovirus, is typically spread by Aedes mosquitoes amongst humans and nonhuman primates, exhibiting high potential for spillover and spillback pathogenic events (Tsetsarkin et al. 2016). Chikungunya virus has been reportedly found capable of involving sylvatic mosquito species such as Haemagogus leucocelaenus and Aedes terrens for pathogenic transmission (Weaver 2014). Several Aedes species have been found to be enzootic transmitters of the disease. These vectors may be responsible for disease transmission between non-human primates and other vertebrates serving as amplification hosts (Tsetsarkin et al. 2016). Data confirming cross species transmission or anthroponosis with regards to vector borne Chikungunya virus is scarce, however in experimental studies $A$. furcifer has successfully transmitted virus from infected humans to African green monkeys (Mcintosh et al. 1964). Moreover, Senegalese baboons (Monlun et al. 1993) and Congolese chimpanzees (Osterrieth et al. 1960) have also been found seropositive for this virus without clinical manifestation of any malady. Seroprevalence of this virus in non-human primates of Bahia state residing in periurban areas also suggests Reverse zoonosis (Forrester et al. 2012; Ndenga et al. 2017).

\section{Dengue Fever}

Dengue virus belongs to genus Flavivirus, which is also known as an arbovirus. Mosquito, such as Aedes spp., are vectors responsible for spreading this virus. Not unlike other arboviruses, Dengue virus and its reservoir vector concurrently arrived the Americas probably as a consequence of slave trade (Hanley et al. 2013). In 2009, a French study undertaken at one of its colonial territories in Guiana reported endemicity and seroprevalence of Dengue virus types 1 to 4 amongst all kinds of mammalian hosts including primates, rodents, bats and marsupials (Fouque et al. 2006). The rationalization for suggesting cross carrying of viral strains between sylvatic and urban vectors has been reinforced by studies conducted in Brazil, Ecuador, Peru and Colombia, where a common backyard mosquito Aedes albopictus found in urban and peri-urban areas was identified as a carrier for Dengue virus type 3 (Usme-Ciro et al. 2008). Likewise, Haemogogus leucocelaenus, a mosquito purely feeding upon wildlife, was discovered to carry Dengue virus type 1 in the state of Bahia (De Figueiredo et al. 2010). Presence of antibodies has also been used as an indicator to confirm the presence of sylvatic lifecycles in the case of Dengue virus. A study in the Sapajus xanthosternos and Leontopithecus chrysomelas primates indigenous to Atlantic forest of Bahia revealed the presence of antibodies against Dengue virus type 1 and 2 (Catenacci et al. 2018). Similarly, Dengue virus type 3 antibodies have been reported in Sloths (Bradypus torquatus) as well (Catenacci et al. 2018). 


\section{Influenza Virus}

There have been reported cases of Influenza A virus subtype $\mathrm{H}_{1} \mathrm{~N}_{1}$ being transmitted from humans to wild, companion and domesticated mammals due to contact through abiotic elements of environment (de Jong and Hien 2006; Morens and Taubenberger 2010; Messenger et al. 2014). This phenomenon is justified by the fact that the said virus is extremely resilient and capable of mass infections without any reservoir host or biological vector (Zambon 2014). Most viruses have a narrow host range, however, antigenic shift, drift and point mutations could be responsible for cross species infections (Zimmer and Burke 2009; Ma et al. 2018). In 1990s, a wild seal admitted to Dutch seal rehabilitation center was reported to be infected with human influenza B virus (Osterhaus et al. 200o). The close proximity, with humans cohabiting in close quarters with companion animals, increase the probability of Reverse zoonosis several folds, especially in case of Influenza virus that may be transmitted through contact with soft, porous surfaces (Mukherjee et al. 2012). An incidence confirming this deduction was reported in 2009, when household pet ferrets were naturally infected with human $\mathrm{H}_{1} \mathrm{~N}_{1}$ strain (Swenson et al. 2010). Viral infections jumping across species are not exclusively seen only in companion animals, but several studies have reported such incidences in livestock as well (Janke 2014). However, the underlying factors, such as contact with contaminants, remain the primary cause of such instances. In Norway, a flock of turkeys contracted Influenza type A HiN1 virus from a diseased farm hand. Phylogenetic analysis of isolates from both species revealed identical set of strains infecting both of them (Kulberg Sjurseth et al. 2017). The transmission of $\mathrm{H}_{1} \mathrm{~N}_{1}$ from humans to pigs is extremely widespread and cases have been identified throughout the South-East Asia (Song et al. 2010), Europe (Chastagner et al. 2019) and Canada (Howden et al. 2009).

\section{Coronavirus}

Wild animals in captivity and sanctuaries have reportedly been victims of Reverse zoonosis, especially in the case of coronavirus. Contrary to popular belief, coronavirus existed before COVID-19 outbreak in both humans and animal species ( $\mathrm{Ng}$ and Hiscox 2020). Previously, symptoms and genetics of coronaviruses afflicting human and animal hosts were distinct. However, it was vexing when coronavirus isolates from a 2007 outbreak in Alpacas was evolutionarily quite similar to that of human coronavirus. This discovery has suggested the human to alpaca mode of transmission. Moreover, these findings have become succinctly significant in the light of recent events when a genetic relative of the same coronavirus is wreaking havoc on human population (Crossley et al. 2012). In 2016, a similar instance of human coronavirus (HCoV-OC43) transmission to wild chimpanzees housed at Taï National Park, Côte d'Ivoire was reported to cause common cold (Mackay et al. 2012). Through the years, coronavirus has been detected in all classes of the animals, ranging from ungulates to carnivores, iterating plausibility of coronavirus host switching (Patrono et al. 2018).

\section{COVID-19}

Recent COVID-19 pandemic has caused scientists to investigate the ability of household pets to serve as carriers or reservoirs of this virus. Experimentation has suggested the transmission and clinical incidence of the disease in cats and ferrets (Shi et al. 2020). The water droplets contaminated with SARS-COV-19 virus can be transmitted to cats. The fecal samples from inoculated cats exhibited viral shedding within 3-5 days. Moreover, viral RNA samples were also collected from upper and lower respiratory tract. Severe lesions were observed in lungs of kittens and mucosae of upper respiratory tract (Shi et al. 2020). Some instances of natural transmissions were reported in domesticated cats, while most experimental investigations were conducted in laboratory settings (Bosco-Lauth et al. 2020). In addition to cats, ferrets have also been observably prone to reverse zoonosis on account of COVID-19 virus (Monchatre-Leroy et al. 2021). In experimental settings, ferrets were inoculated with samples obtained from Huanan Seafood Market in Wuhan, China and clinically positive humans causing subsequent shedding of virus. Virus successfully replicated in the upper respiratory tract mucosae and remained detectable in rectal samples for prolonged period without causing death. Thirteen days post inoculation of viral isolates, mild peri-bronchitis along with other marked symptoms associated with COVID-19 were observed. Serum antibodies against SARS-CoV-2 were also detected (Shi et al. 2020). Contrarily, low susceptibility was seen after inoculation of virus into Beagle dogs whereby, only $50 \%$ of them seroconverted after 14 days (Shi et al. 2020). However, such findings were not observed when chicken, ducks and pigs were inoculated, as these species remained seronegative even 14 days after infection (Shi et al. 2020).

\section{Measles}

Measles is a common pediatric infection of viral origin caused by measles virus $(\mathrm{MeV})$, which is a member of the genus Morbillivirus. Phylogenetic evidence has suggested that $\mathrm{MeV}$ has evolved from Rinderpest virus (RPV) around the 11th to the 12th century and is commonly associated with infections in cattle (Furuse et al. 2010). Since then, measles has wreaked havoc on human populations, causing high morbidity and mortality in infected individuals (Hilleman 1992). The outbreak reported in 1996 amongst 94 primates has since then been identified as an instance of Reverse zoonosis following urine and serum analysis were found positive for measle specific IgG and IgM antibodies. A reverse transcriptase-polymerase chain reaction was also employed for confirmatory virus isolation (Willy et al. 1999). The incidence of measles outbreak in humans precisely during the same period legitimized the said claims (Willy et al. 1999). 


\section{Rhinovirus-C}

Rhinoviruses, which belong to the family Picornaviridae and genus Enteroviridae, are responsible for causing common cold in humans (Matsuzawa 2020). Interestingly, human rhinovirus-3 was never thought to be capable of jumping species. However, several reports in non-human primates withsevere flu-like symptoms have indicated a spillover effect into sylvatic communities (Velayudhan et al. 2006). Outbreak of human rhinovirusC (HRV-C) in 2013 amongst chimpanzees has been identified by the presence of universal homozygosity for the $3 \mathrm{CDHR}_{3}-\mathrm{Y}_{529}$ allele (cadherin related family member), which is a receptor that drastically increases susceptibility to rhinovirus- $C$ infection and asthma in humans. Susceptibility of chimpanzees to a highly pathogenic virus implicates inevitable spillback into human hosts (Scully et al. 2018).

\section{Pneumo Viruses}

Human metapneumovirus (also known as MPV, Pneumoviridae, Metapneumovirus) and a human respirovirus-3 (also known as $\mathrm{HRV}_{3}$, Paramyxoviridae, Respirovirus, or formerly as parainfluenza virus-3) have been found as causative agents for spread of respiratory infection amongst Ugandan Chimpanzee community (Negrey et al. 2019).

Human respiratory viruses have become a major threat to wild non-human primate communities (Dunay et al. 2018). These respiratory maladies have become predominant causes of the disease and mortality amongst apes in wildlife sanctuaries of Uganda (Emery Thompson et al. 2018) and Tanzania (Lonsdorf et al. 2018). Reverse zoonosis of human respiratory pneumoviruses has also been reported in gorillas from Congo (Grützmacher et al. 2018) and Central African Republic (Grützmacher et al. 2016).

\section{Parasitic and Fungal Anthroponosis}

In a similar fashion of other infectious agents, parasites and fungi also make their way between humans and animals. Most of the time parasitic cycle of transmission is slower than that of bacteria and viruses due to involvement of intermediate transporters and nature of slow growth in case of parasites. However, parasites are able to share different hosts, including humans and animals. The fungal kingdom contains as many as 6 million species and is important in terms of the breadth and depth of its effects in areas of public health, animal health, environment, biomedical and agriculture (Taylor et al. 2014). Fungi can be naturally transmitted between animals and humans in both directions and in some cases cause significant public health problems. A large number of fungi associated with zoonotic and reverse zoonosis transmission are among the group of the most common fungal diseases worldwide. It is, however, notable that some fungal diseases have failed to attract sufficient attention in international one health efforts, leading to insufficient attention on their preventive strategies (Seyedmousavi et al. 2015).

\section{Cryptosporidium parvum}

A parasitic disease in the intestinal tract of mammals, called cryptosporidiosis, is caused by Cryptosporidium (C.) parvum. The infection of $C$. parvum is mainly manifested by watery, non-bloody acute diarrhea. The presence of cryptosporidiosis disease in livestock has become a considerable glitches both for animal wellbeing (together clinical and subclinical) and commercial harms (Santín 2013). These effects are due to growing veterinary amenities and work expenses, aggregate animal healthcarefulness rate, and deteriorating the growth of animals along with high mortality. Cryptosporidium parvum was one of the most prevalent parasitic species in animals, infecting 30\% of buffaloes at farm level (Inpankaew et al. 2014).

The parasite can be transmitted by two routes, including direct and indirect methods of transmission. Direct transmission occurs through feco-oral route by unintended ingestion that leads to the excretion of Cryptosporidium oocytes in feces. A parasite adopts various routes for its transmission from humans to animals or vice versa and for human to human transmission (Hubalek 2003). In humans, it causes infection by living in the epithelial exteriors of the intestines, distributed from stool or feces, and/or filthy in the atmosphere (environmental contamination). There are only a few studies for animals that demonstrate the influence of nitazoxanide drug contrary to clinical contaminations of Cryptosporidium spp. in which this nitazoxanide drug might reduce Cryptosporidium oocysts in defecation. Nevertheless, this drug is not being used commonly in animals (Ghazy et al. 2015). There is an extensive need to practice the preventive measures to reduce the spread of Cryptosporidium spp. particularly in livestock. By mitigating different factors, namely congestion in farmhouses, cross contamination by workers and contact with animals which bear greater risk of infection (Robertson et al. 2020).

\section{Trichuris trichiura}

Trichuris (T.) trichiura is a species that can be found in the tropical and temperate areas around the globe, however, mostly found in the dampness of the tropics (Roberts and Janovy Jr, 200o). This parasitic specie is also named as whipworm and infects animals and humans. There are two situations, namely deprived sanitation and poor environment, required for dispersion of T. trichiura and for the development of worm. These environmental conditions comprise a moisturizing moderate climate, wet soil, low lights and lot of rainfall (de Silva et al. 2003). The eggs of $T$. trichiura have been detected in the feces of some pigs, dogs and cats in tropical areas with poor sanitation, raising the possibility of reverse zoonoses. T. trichiura was also identified in mountain gorillas, and there are possibilities that these parasites 
belong to human origin (Sleeman et al. 200o). A recent study in Thailand found that more than half of the Trichuris-positive fecal samples from dogs contained the human parasite $T$. trichiura, as identified by PCR. Likewise, more than half of the fecal samples from dogs and cats in Malaysia were found to contain eggs identified genetically as from T. trichiura. Whether these animals were infected with adult worms or simply shedding eggs acquired from the environment is currently unclear. (Phosuk et al. 2018; Mohd-Shaharuddin et al. 2019).

\section{Blastocystis spp}

Blastocystis is a genus in the Stramenopile group that is very diverse, comprising brown algae, slime nets, diatoms and group of water molds. These Blastocystis are eukaryotic organisms that are unicellular and anaerobic in nature (Hoevers and Snowden 2005). It is the most common parasitic protest globally and also a pluralistic enteric parasite found worldwide (Ahmed and Karanis 2018). The virulence mode of action of Blastocystis is not fully described yet. Although, it has pathogenic potential but its capability to cause gastrointestinal and various other infections has been questioned (Mohamed et al. 2017). A vast variety of animals, such as invertebrates, reptiles, amphibians, birds, mammals and humans, are infected by Blastocystis (Ahmed and Karanis 2018). One of the most common parasites that infect humans globally is the Blastocystis, as it is extensively spread worldwide. The commonly acknowledged pathway of transmission is the feco-oral diffusion and current investigations have revealed that spread occurs merely through the cyst form of the parasite (Yoshikawa et al. 2004). However, when ingestion of the cyst takes place, its life cycle is started. Different forms of cyst develop after ingestion, which possibly will turn into cyst forms sequentially. The transmission cycle is repeated when cysts are released into environment through humanoid feces to infect the humans and other animals. The contamination caused by Blastocystis parasites can take place by different species either through zoonosis or reverse zoonosis (Noël et al. 2005). However, studies found zoonotic isolates having large potential and stay problematic in recognizing the host roots and communication ways. Blastocystis, whether commensal, pathogen, or part of human microbiota, is still ubiquitous parasite cycling among humans, animals and environment. Evolution of this parasite is increasingly reported almost everywhere worldwide involving animals, humans, and even water. However, Blastocystis still remains underestimated and underreported in comparison to other protozoa (Ahmed and Karanis 2018).

\section{Encephalitozoon intestinalis}

Encephalitozoon (E.) intestinalis is one of the most predominant Encephalitozoon species found in humans around the world. This specie has been found to occur in numerous, principally native, mammalian species, for example cattle, pigs, goats, donkeys, dogs or gorillas
(Hinney et al. 2016). Animal to human and human to animal transmission has been proposed to be an important source of infection. Human associated Encephalitozoon spp. was found in waterfowl in Slovikia (Malčeková et al. 2013). Several case reports of this parasite transmission in captive wild birds have been described. Subsequent studies on larger populations showed that asymptomatic infections in captive wild birds are widespread (Phalen et al. 2006). Close interaction between humans and non-human primates can create routes for the transmission of this zoonotic disease in both directions (Graczyk et al. 2002).

\section{Candida albicans}

Species of Candida, a yeast like fungus, cause a localized mucocutaneous disease called "Candidiasis" (Hani et al. 2015). Candidiasis is prevalent all over the world in humans and animals. Many animal cases were reported that had the same isolates as those of humans (Wrobel et al. 2008). Canine isolate was obtained from a graduate student-owned dog and two feline isolates were obtained from animals at the Champaign County Humane Society analysis of the domestic animals. Candida albicans isolates showed that the cats and dogs were colonized with common human isolates. The pets tested were all indoor animals that were in close contact with humans (Wrobel et al. 2008). Parakeet (bird) was found infected with $C$. albicans. It was also a case of reverse zoonosis (Kumar et al. 2017). Ingestion of contaminated food or drinking water is the usual means of transmission. Contaminated environment, such as litter from diseased and game bird rearing in the same amenities and areas contaminated with human waste, are suggested as sources for Candidiasis exposure to birds (Kumar et al. 2017). The consensus drawn from various texts is that $C$. albicans is associated mainly with humans, other mammals, and avian species (Wrobel et al. 2008).

\section{Trichophyton rubrum}

Trichophyton (T.) rubrum belongs to phylum Ascomycota. It is dermatophytic, anthropophilic, saprotrophic fungus and exclusively clonal, which colonizes the upper layers of dead skin cells (Gräser et al. 1999). Trichophyton rubrum is a cosmopolitan, anthropophilic species and is the most usual causative agent of human dermatophytosis across the planet. Despite being anthropophilic, this dermatophyte is also infrequently found to be reason of dermatological disease in dogs. Therefore, T. rubrum can also be considered as an anthropozoonotic and zooanthroponotic pathogen (Overgaauw et al. 2020).

\section{Microsporum gypseum}

Microsporum gypseum is a soil-associated dermatophyte that is recognized to colonize and infect the upper layer of skin in mammals (Samanta, 2015). Tinea or ringworm is the fungal infection, which affects external body parts. It 
is one of the fungal diseases which are transferred from humans to animals and humans to humans (Summerbell and Howard, 2003). People engaged in experimentation and handling with laboratory animals can also be at risk of contracting the infection, if the animals were predisposed to a certain region (Ranganathan and Balajee 200o). Animals such as cattle, which repeatedly come in contact with soil, are predominantly affected by this fungus, but equines, rodents, apes and canines have a disposition to acquire it (Messenger et al. 2014). A dog was found positive with M. gypseum, which is a case of zooanthroponosis (Sharma et al. 2009); though the soil is usually considered as the prime reason of infection. Analytically, at least 10 fungal spores must deposit on a keratin rich skin surface of animals or humans to manifest a fungal infection (Messenger et al. 2014). Diagnosis is usually made on the shape of the fungus on the host through a sample culture.

\section{Conclusion}

Reverse Zoonosis is a reality and is playing an important role in the sufferings of the animals. During the past few decades, human animal interaction, especially with the wild life, has increased. Transmission of interspecies pathogens could be direct or through vectors. All types of pathogens i.e., bacteria, viruses, protozoa, and fungi have capability of reverse zoonosis. Although it is difficult and labor extensive work to consider animal factors while studying a disease outbreak in humans, yet it is need of the time, as the evidences are strong for inter species disease transmission. The countries having higher population density like Pakistan, have higher chances of human to animal transmission of pathogens, as they are residing in close vicinity. There is need to focus on this aspect of research and study in Pakistan. In the light of COVID-19 disaster, the thinking horizon of the scientist has now changed. There will be more focus on 'One Health' for one globe rather than only human health. Because health is important, host specie doesn't matter now.

\section{REFERENCES}

Adesokan HK et al., 2012. Mycobacterium bovis infection in livestock workers in Ibadan, Nigeria: evidence of occupational exposure. The International Journal of Tuberculosis and Lung Disease 16: 1388-1392.

Ahmed SA and Karanis P, 2018. Blastocystis spp., Ubiquitous Parasite of human, animals and environment. Encyclopedia of Environmental Health, 2nd Edition. Elsvier.

Alekseev A and Dubinina H, 2001. Bloodsucking arthropods: the danger for travellers and hazard of vector travelling. Wiadomości Parazytologiczne 47: 33-37.

Armand-Lefevre L et al., 2005. Clonal comparison of Staphylococcus aureus isolates from healthy pig farmers, human controls, and pigs. Emerging Infectious Diseases 11: 711-714.
Asiak IE et al., 2007. The use of ELISA in the detection of bovine tuberculosis in slaughtered cattle and sedentary herds in South Western Nigeria. Journal of Animal and Veterinary Advances 7: 883-886.

Barasona JA et al., 2017. Environmental presence of Mycobacterium tuberculosis complex in aggregation points at the wildlife/livestock interface. Transboundary and Emerging Diseases 64: 1148-1158.

Bayraktar B et al., 2011a. Species distribution of the Mycobacterium tuberculosis complex in clinical isolates from 2007 to 2010 in Turkey: a prospective study. Journal of Clinical Microbiology 49:3837-3841.

Bayraktar B et al., 2011b. Mycobacterium caprae causing lymphadenitis in a child. The Pediatric Infectious Diseases Journal 30: 1012-1013.

BhanuRekha V et al., 2015. Molecular detection of Mycobacterium tuberculosis from bovine milk samples. Journal of Advanced Veterinary and Animal Research 2(1): 80-83.

Bosco-Lauth AM et al., 2020. Experimental infection of domestic dogs and cats with SARS-CoV-2: Pathogenesis, transmission, and response to reexposure in cats. Proceedings of the National Academy of Sciences, USA 117: 26382-26388.

Catenacci LS et al., 2018. Surveillance of arboviruses in primates and sloths in the Atlantic Forest, Bahia, Brazil. EcoHealth 15: 777-791.

Chastagner A et al., 2019. Bidirectional human-swine transmission of seasonal influenza $\mathrm{A}\left(\mathrm{H}_{1} \mathrm{~N}_{1}\right)$ pdmo9 virus in pig herd, France, 2018. Emerging Infectious Diseases 25: 1940.

Chippaux J-P and Chippaux A, 2018. Yellow fever in Africa and the Americas: a historical and epidemiological perspective. Journal of Venomous Animals and Toxins including Tropical Diseases 24: 20.

Coffey LL et al., 2017. Final Report May 22, 2017.

Coque TM et al., 2008. Increasing prevalence of ESBLproducing Enterobacteriaceae in Europe. Eurosurveillance 13: 19044.

Crossley BM et al., 2012. Identification and characterization of a novel alpaca respiratory coronavirus most closely related to the human coronavirus 229E. Viruses 4: 3689-3700.

Dunay E et al., 2018. Pathogen transmission from humans to great apes is a growing threat to primate conservation. EcoHealth 15: 148-162.

Emery Thompson $M$ et al., 2018. Risk factors for respiratory illness in a community of wild chimpanzees (Pan troglodytes schweinfurthii). Royal Society Open Science 5: 180840.

Every AL et al., 2011. Did transmission of Helicobacter pylori from humans cause a disease outbreak in a colony of Stripe-faced Dunnarts (Sminthopsis macroura)? Veterinary Research 42: 26.

Ewers $\mathrm{C}$ et al., 2010. Emergence of human pandemic $\mathrm{O}_{25}: \mathrm{H}_{4}-\mathrm{ST}_{131}$ CTX-M-15 extended-spectrum-betalactamase-producing Escherichia coli among companion animals. The Journal of Antimicrobial Chemotherapy 65: 651-66o. 
Fernandes NCC de A et al., 2017. Outbreak of Yellow Fever among nonhuman primates, Espirito Santo, Brazil, 2017. Emerging Infectious Diseases 23: 20382041.

Figueiredo PO et al., 2018. Detection and molecular characterization of Yellow Fever virus, 2017, Brazil. EcoHealth 15: 864-870.

Figueiredo LTM 2019. Human urban arboviruses can infect wild animals and jump to sylvatic maintenance cycles in South America. Frontiers in Cellular and Infection Microbiology 9: 259.

De Figueiredo ML et al., 2010. Mosquitoes infected with dengue viruses in Brazil. Virology Journal 7: 1-5.

Fluit AC 2012. Livestock-associated Staphylococcus aureus. Clinical Microbiology and Infection 18: 735-744.

Forrester NL et al., 2012. Genome-scale phylogeny of the alphavirus genus suggests a marine origin. Journal of Virology 86: 2729-2738.

Fouque $\mathrm{F}$ et al., 2006. Aedes aegypti survival and dengue transmission patterns in French Guiana. Journal of Vector Ecology 31: 390-399.

Furuse $\mathrm{Y}$ et al., 2010. Origin of measles virus: divergence from rinderpest virus between the 11th and 12th centuries. Virology Journal 7: 1-4.

Ghazy AA et al., 2015. Cryptosporidiosis in animals and man: 2. Diagnosis. Asian Journal of Epidemiology 8: 84 .

Goenaga $\mathrm{S}$ et al., 2012. Isolation of yellow fever virus from mosquitoes in Misiones province, Argentina. Vectorborne and Zoonotic Diseases 12: 986-993.

Graczyk TK et al., 2002. A single genotype of Encephalitozoon intestinalis infects free-ranging gorillas and people sharing their habitats in Uganda. Parasitology Research 88: 926-931.

Gräser Y et al., 1999. Molecular markers reveal exclusively clonal reproduction in Trichophyton rubrum. Journal of Clinical Microbiology 37: 3713-3717.

Graveland $\mathrm{H}$ et al., 2011. Livestock-associated methicillinresistant Staphylococcus aureus in animals and humans. International Journal of Medical Microbiology 301: 630-634.

Grützmacher KS et al., 2016. Codetection of Respiratory Syncytial Virus in habituated wild western lowland gorillas and humans during a respiratory disease outbreak. EcoHealth 13: 499-510.

Grützmacher KS et al., 2018. Human Respiratory Syncytial Virus and Streptococcus pneumoniae infection in wild bonobos. EcoHealth 15: 462-466.

Hackendahl NC et al., 2004. Putative transmission of Mycobacterium tuberculosis infection from a human to a dog. Journal of the American Veterinary Medical Association 225: 1573-1577.

Hamrick PN et al., 2017. Geographic patterns and environmental factors associated with human yellow fever presence in the Americas. PLoS Neglected Tropical Diseases 11: eooo5897.

Hani U et al., 2015. Candidiasis: a fungal infection-current challenges and progress in prevention and treatment. Infectious Disorders Drug Targets 15: 4252.
Hanley KA et al., 2013. Fever versus fever: the role of host and vector susceptibility and interspecific competition in shaping the current and future distributions of the sylvatic cycles of dengue virus and yellow fever virus. Infection, Genetics and Evolution 19: 292-311.

Hasman $\mathrm{H}$ et al., 2010. Spa type distribution in Staphylococcus aureus originating from pigs, cattle and poultry. Veterinary Microbiology 141: 326-331.

Hilleman MR 1992. Past, present, and future of measles, mumps, and Rubella virus vaccines. Pediatrics 90: 149-153.

Hinney B et al., 2016. More than a rabbit's taleEncephalitozoon spp. in wild mammals and birds. International Journal for Parasitology: Parasites and Wildlife 5: 76-87.

Hoevers JD and Snowden KF, 2005. Analysis of the ITS region and partial ssu and lsu rRNA genes of Blastocystis and Proteromonas lacertae. Parasitology 131: 187-196.

Howden $\mathrm{KJ}$ et al., 2009. An investigation into human pandemic influenza virus $\left(\mathrm{H}_{1} \mathrm{~N}_{1}\right) 2009$ on an Alberta swine farm. The Canadian Veterinary Journal 50: 1153.

Hubálek Z 2003. Emerging human infectious diseases: anthroponoses, zoonoses, and sapronoses. Emerging Infectious Diseases 9: 403.

Inpankaew $\mathrm{T}$ et al., 2014. Molecular detection of Cryptosporidium spp. infections in water buffaloes from northeast Thailand. Tropical Animal Health and Production 46: 487-490.

Janke BH 2014. Influenza A virus infections in swine: pathogenesis and diagnosis. Veterinary Pathology 51: 410-426.

Jenkins AO et al., 2011. Molecular epidemiology of human and animal tuberculosis in Ibadan, Southwestern Nigeria. Veterinary Microbiology 151: 139-147.

Joint FAO and Organization WH, 1967. Joint FAO/WHO Expert Committee on Zoonoses [meeting held in Geneva from 6 to 12 December 1966]: third report.

de Jong MD and Hien TT, 2006. Avian influenza A $\left(\mathrm{H}_{5} \mathrm{~N}_{1}\right)$. Journal of Clinical Virology 35: 2-13.

Köck $\mathrm{R}$ et al., 2009. Prevalence and molecular characteristics of methicillin-resistant Staphylococcus aureus (MRSA) among pigs on German farms and import of livestock-related MRSA into hospitals. European Journal of Clinical Microbiology and Infectious Diseases 28: 1375-1382.

Köck $\mathrm{R}$ et al., 2013. Livestock-associated methicillinresistant Staphylococcus aureus (MRSA) as causes of human infection and colonization in Germany. PloS One 8: e55040-e55040.

Kulberg Sjurseth S et al., 2017. Human to animal transmission of influenza A $\left(\mathrm{H}_{1} \mathrm{~N}_{1}\right)$ pdmog in a turkey breeder flock in Norway. Infection Ecology and Epidemiology 7: 1416249.

Kumar SK et al., 2017. Candidiasis in a parakeet-an avenue to zooanthroponosis. Journal of Animal Health and Production 5: 85-88.

Kuno $G$ et al., 1998. Phylogeny of the genus Flavivirus. Journal of Virology 72: 73-83. 
LeBreton $\mathrm{M}$ et al., 2007. Exposure to wild primates among $\mathrm{HIV}$-infected persons. Emerging Infectious Diseases 13: 1579.

Lonsdorf EV et al., 2018. Socioecological correlates of clinical signs in two communities of wild chimpanzees (Pan troglodytes) at Gombe National Park, Tanzania. American Journal of Primatology 8o(1): 22562. doi: 10.1002/ajp.22562.

Ma MJ et al., 2018. Evidence for cross-species influenza A virus transmission within swine farms, China: a one health, prospective cohort study. Clinical Infectious Diseases 66: 533-540.

Mackay IM et al., 2012. Co-circulation of four human coronaviruses (HCoVs) in Queensland children with acute respiratory tract illnesses in 2004. Viruses 4: 637-653.

Malčeková B et al., 2013. First detection and genotyping of human-associated microsporidia in wild waterfowl of Slovakia. Acta Parasitologica 58: 13-17.

Matsuzawa $\mathrm{T}$ 2020. Jokro: The death of a wild infant chimpanzee from respiratory disease. Primates 61: 339-346.

Mcintosh BM et al., 1964. Further studies on the chikungunya outbreak in Southern Rhodesia in 1962. Annals of Tropical Medicine and Parasitology 58: 4551.

Messenger AM et al., 2014. Reverse zoonotic disease transmission (zooanthroponosis): a systematic review of seldom-documented human biological threats to animals. PloS One 9: e89055.

Miller MA et al., 2019. Fatal tuberculosis in a free-ranging African elephant and one health implications of human pathogens in wildlife. Frontiers in Veterinary Science 6: 18 .

Moda G et al., 1996. The zoonotic importance of Mycobacterium bovis. Tubercle and lung disease. The Official Journal of the International Union against Tuberculosis and Lung Disease 77: 103-108.

Mohamed AM et al., 2017. Predominance and association risk of Blastocystis hominis subtype I in colorectal cancer: a case control study. Infectious Agents and Cancer 12: 21.

Mohd-Shaharuddin N et al., 2019. Molecular characterization of Trichuris species isolated from humans, dogs and cats in a rural community in Peninsular Malaysia. Acta Tropica 190: 269-272.

Monath TP and Vasconcelos PFC, 2015. Yellow fever. Journal of Clinical Virology 64: 160-173.

Monchatre-Leroy E et al., 2021. Hamster and ferret experimental infection with intranasal low dose of a single strain of SARS-CoV-2. Journal of General Virology 102(3): 1567. doi: 10.1099/jgv.0.001567.

Monlun E et al., 1993. Surveillance of the circulation of arbovirus of medical interest in the region of eastern Senegal. Bulletin de la Societe de Pathologie Exotique (1990) 86: 21-28.

Morens DM and Taubenberger JK, 2010. Historical thoughts on influenza viral ecosystems, or behold a pale horse, dead dogs, failing fowl, and sick swine. Influenza and Other Respiratory Viruses 4: 327-337.
Mukherjee DV et al., 2012. Survival of influenza virus on hands and fomites in community and laboratory settings. American Journal of Infection Control 40: 590-594.

Mundy NI et al., 1998. Protein deficiency in a colony of western lowland gorillas (Gorilla g. gorilla). Journal of Zoo and Wildlife Medicine 29: 261-268.

Nah K et al., 2016. Estimating risks of importation and local transmission of Zika virus infection. Peer $\mathrm{J} 4$ : e1904.

Ndenga BA et al., 2017. Characteristics of Aedes aegypti adult mosquitoes in rural and urban areas of western and coastal Kenya. PloS One 12: eo189971.

Negrey JD et al., 2019. Simultaneous outbreaks of respiratory disease in wild chimpanzees caused by distinct viruses of human origin. Emerging Microbes and Infections 8: 139-149.

Nelson MI and Vincent AL 2015. Reverse zoonosis of influenza to swine: new perspectives on the humananimal interface. Trends in Microbiology 23: 142-153.

$\mathrm{Ng}$ LFP and Hiscox JA, 2020. Coronaviruses in animals and humans. British Medical Journal 368.

Nicolas-Chanoine $\mathrm{M}-\mathrm{H}$ et al., 20o8. Intercontinental emergence of Escherichia coli clone $\mathrm{O}_{25}: \mathrm{H}_{4}-\mathrm{ST}_{131}$ producing CTX-M-15. The Journal of Antimicrobial Chemotherapy 61: 273-281.

Nizeyi JB et al., 2001. Campylobacteriosis, salmonellosis, and shigellosis in free-ranging human-habituated mountain gorillas of Uganda. Journal of Wildlife Diseases 37: 239-244.

Noël C et al., 2005. Molecular phylogenies of Blastocystis isolates from different hosts: implications for genetic diversity, identification of species, and zoonosis. Journal of Clinical Microbiology 43: 348-355.

Ocepek M et al., 2005. Transmission of Mycobacterium tuberculosis from human to cattle. Journal of Clinical Microbiology 43: 3555-3557.

Osterhaus A et al., 200o. Influenza B virus in seals. Science 288: 1051-1053.

Osterrieth P et al., 1960. Research on the Chikungunya virus in the Belgian Congo. II. Serological investigation. Annales de la Societe Belge de Medecine Tropicale (1920) 40: 205-213.

Overgaauw PAM et al., 2020. A one health perspective on the human-companion animal relationship with emphasis on zoonotic aspects. International Journal of Environmental Research and Public Health 17: 3789 .

Patrono LV et al., 2018. Human coronavirus $\mathrm{OC}_{43}$ outbreak in wild chimpanzees, Cote d Ivoire, 2016. Emerging Microbes and Infections 7: 1-4.

Phalen DN et al., 2006. Encephalitozoon hellem infection as the cause of a unilateral chronic keratoconjunctivitis in an umbrella cockatoo (Cacatua alba). Veterinary Ophthalmology 9: 59-63.

Phosuk I et al., 2018. Molecular identification of Trichuris suis and Trichuris trichiura eggs in human populations from Thailand, Lao PDR, and Myanmar. The American Journal of Tropical Medicine and Hygiene 98: 39-44. 
Pomba $\mathrm{C}$ et al., 2009. Detection of the pandemic O25ST131 human virulent Escherichia coli CTX-M-15producing clone harboring the qnrB2 and aac(6')-Ibcr genes in a dog. Antimicrobial Agents and Chemotherapy 53: 327-328.

Price LB et al., 2012. Staphylococcus aureus CC398: host adaptation and emergence of methicillin resistance in livestock. mBio 3(1): eoo305-11.

Ranganathan S and Balajee SA, 200o. Microsporum gypseum complex in Madras, India. Mycoses 43: 177180.

Roberts L and Janovy Jr J, 200o. Foundations of Parasitology . $8^{\text {th }}$ Edition New York.

Robertson LJ et al., 2020. Cryptosporidium infections in Africa-How important Is zoonotic transmission? A review of the evidence. Frontiers in Physiology 7: 575881.

Rwego IB et al., 2008. Gastrointestinal bacterial transmission among humans, mountain gorillas, and livestock in Bwindi Impenetrable National Park, Uganda. Conservation Biology: the Journal of the Society for Conservation Biology 22: 1600-1607.

Samanta I, 2015. Veterinary Mycology. Springer.

Santín M, 2013. Clinical and subclinical infections with Cryptosporidium in animals. New Zealand Veterinary Journal 61: 1-10.

Schaumburg F et al., 2012. Drug-resistant human Staphylococcus aureus in sanctuary apes pose a threat to endangered wild ape populations. American Journal of Primatology 74: 1071-1075.

Scully EJ et al., 2018. Lethal respiratory disease associated with human rhinovirus $\mathrm{C}$ in wild chimpanzees, Uganda, 2013. Emerging Infectious Diseases 24: 267.

Seguin JC et al., 1999. Methicillin-resistant Staphylococcus aureus outbreak in a veterinary teaching hospital: potential human-to-animal transmission. Journal of Clinical Microbiology 37: 1459-1463.

Seyedmousavi S et al., 2015. Neglected fungal zoonoses: hidden threats to man and animals. Clinical Microbiology and Infection 21: 416-425.

Sharma DK et al., 2009. Zooanthroponosis of Microsporum gypseum infection. Haryana Veterinarian 48: 108-109.

Shepheard MA et al., 2013. Historical zoonoses and other changes in host tropism of Staphylococcus aureus, identified by phylogenetic analysis of a population dataset. PLoS One 8: e62369.

Shi J et al., 2020. Susceptibility of ferrets, cats, dogs, and other domesticated animals to SARS-coronavirus-2. Science 368: 1016-1020.

Shitaye JE et al., 2007. Bovine tuberculosis infection in animal and human populations in Ethiopia. Veterinární Medicína 52: 317-332.

de Silva NR et al., 2003. Soil-transmitted helminth infections: updating the global picture. Trends in Parasitology 19: 547-551.

Singh RK et al., 2016. Zika virus-emergence, evolution, pathology, diagnosis, and control: current global scenario and future perspectives-a comprehensive review. Veterinary Quarterly 36: 150-175.
Sleeman JM et al., 20oo. Gastrointestinal parasites of mountain gorillas (Gorilla gorilla beringei) in the PARC National des Volcans, Rwanda. Journal of Zoo and Wildlife Medicine 31: 322-328.

Smith TC 2015. Livestock-associated Staphylococcus aureus: the United States experience. PLoS Pathogens 11: e1004564.

Song MS et al., 2010. Evidence of human-to-swine transmission of the pandemic $\left(\mathrm{H}_{1} \mathrm{~N}_{1}\right) 2009$ influenza virus in South Korea. Journal of Clinical Microbiology 48: 3204-3211.

Spoor LE et al., 2013. Livestock origin for a human pandemic clone of community-associated methicillin-resistant Staphylococcus aureus. mBio 4: eoo356-13.

Stetter MD et al., 1995. Shigellosis in captive western lowland gorillas (Gorilla gorilla gorilla). Journal of Zoo and Wildlife Medicine 26: 52-6o.

Summerbell R and Howard DH, 2003. Pathogenic fungi in humans and animals. Ascomycetes: Aspergillus, Fusarium, Sporothrix and Piedraia and Their Relatives. 2nd Edition. New York: Marcell Decker 237-498.

Sung JM-L et al., 2008. Staphylococcus aureus host specificity: comparative genomics of human versus animal isolates by multi-strain microarray. Microbiology (Reading, England) 154: 1949-1959.

Swenson SL et al., 2010. Natural cases of 2009 pandemic $\mathrm{H}_{1} \mathrm{~N}_{1}$ influenza $\mathrm{A}$ virus in pet ferrets. Journal of Veterinary Diagnostic Investigation 22: 784-788.

Taylor DL et al., 2014. A first comprehensive census of fungi in soil reveals both hyperdiversity and fine-scale niche partitioning. Ecological Monographs 84:3-20.

Tong SYC et al., 2015. Staphylococcus aureus infections: epidemiology, pathophysiology, clinical manifestations and management. Clinical Microbiology and Infection 28: 603-661.

Torgerson PR and Torgerson DJ, 2010. Public health and bovine tuberculosis: what's all the fuss about? Trends in Microbiology 18: 67-72.

Tsetsarkin KA et al., 2016. Interspecies transmission and chikungunya virus emergence. Current Opinion in Virology 16: 143-150.

Usme-Ciro JA et al., 20o8. Simultaneous circulation of genotypes I and III of dengue virus 3 in Colombia. Virology Journal 5: 1-10.

Velayudhan BT et al., 2006. Human metapneumovirus in turkey poults. Emerging Infectious Diseases 12: 18531859 .

Volk SM et al., 2010. Genome-scale phylogenetic analyses of chikungunya virus reveal independent emergences of recent epidemics and various evolutionary rates. Journal of Virology 84: 6497-6504.

Weaver SC, 2014. Arrival of chikungunya virus in the new world: prospects for spread and impact on public health. PLoS Neglected Tropical Diseases 8: e2921e2921.

Willy ME et al., 1999. Management of a measles outbreak among Old World nonhuman primates. Comparative Medicine 49: 42-48. 
Wrobel L et al., 20o8. Molecular phylogenetic analysis of a geographically and temporally matched set of Candida albicans isolates from humans and nonmigratory wildlife in central Illinois. Eukaryotic Cell 7: 1475-1486.

Yen PS et al., 2018. Aedes aegypti mosquitoes from Guadeloupe (French West Indies) are able to transmit yellow fever virus. PLoS One 13: e0204710.

Yoshikawa $\mathrm{H}$ et al., 2004. Fecal-oral transmission of the cyst form of Blastocystis hominis in rats. Parasitology Research 94: 391-396.
Zambon M, 2014. Influenza and other emerging respiratory viruses. Medicine 42: 45-51.

Zhou W et al., 2018. WGS analysis of ST9-MRSA-XII isolates from live pigs in China provides insights into transmission among porcine, human and bovine hosts. Journal of Antimicrobial Chemotherapy 73: 2652-2661.

Zimmer SM and Burke DS, 2009. Historical perspectiveemergence of influenza $A\left(\mathrm{H}_{1} \mathrm{~N}_{1}\right)$ viruses. New England Journal of Medicine 361: 279-285. 